\title{
Comment on: On the irreducibility of the Severi variety of nodal curves in a smooth surface, by E. Ballico
}

\author{
Thomas Dedieu
}

\begin{abstract}
In this short note, I point out that results of Ballico and Kool-Shende-Thomas together imply that on $K 3$, Enriques, and Abelian surfaces, if $L$ is a very ample and $\left(2 p_{a}(L)-2 g-1\right)$-spanned line bundle, then the equigeneric Severi variety $V_{g}(L)$ of all curves in $|L|$ having genus $g$ is non-empty, irreducible, of the expected dimension, and its general member is a $\left(p_{a}(L)-g\right)$-nodal curve.
\end{abstract}

Let $S$ be a smooth, complex, projective surface, and $L$ an effective line bundle on $S$. We denote by $p(L)$ the common arithmetic genus of all members of the linear system $|L|$. For nonnegative integers $g$ and $\delta$, we consider the equigeneric Severi variety $V_{g}(L)$ (resp. nodal Severi variety $V^{\delta}(L)$ ), namely the locally closed subset in $|L|$ corresponding to reduced curves of geometric genus $g$ (resp. with $\delta$ ordinary double points and no further singularity). In particular, $V^{\delta}(L)$ is an open subset of $V_{p(L)-\delta}(L)$.

In the recent paper [1] Ballico has proven that if $L$ is very ample and $(2 \delta-1)$-spanned, then the nodal Severi variety $V^{\delta}(L)$, if non-empty, is irreducible of codimension $\delta$ in $|L|$. Here I show how this result can be enhanced by taking in consideration a former result due to Kool, Shende and Thomas. This text is merely intended as a complement to [1], and I thank Edoardo Ballico for giving me the opportunity to write this up.

Theorem 1. Let $S$ be a $K 3$ (resp. Enriques, resp. Abelian) surface, and $L$ a line bundle on it. Consider an integer $g \leqslant p(L)$. If $L$ is very ample and $(2 p(L)-2 g-1)$-spanned, then the equigeneric Severi variety $V_{g}(L)$ is non-empty and irreducible of dimension $g$ (resp. $g-1$, resp. $g-2)$, and the general member of $V_{g}(L)$ is a nodal curve.

On $K 3$, Enriques, and Abelian surfaces, there are explicit necessary and sufficient conditions for a line bundle to be $k$-spanned, resp. $k$-very ample, $[2,3,13,9]$. In particular, they say that being $k$-spanned and $k$-very ample are two equivalent conditions.

Remark 2. The arguments given here don't ensure that the general member of $V_{g}(L)$ is irreducible. In practice, this may be obtained by studying the various possible splittings of $L$ and a dimension argument.

It is now common knowledge that if $(S, L)$ is a polarized $K 3$ or Abelian surface, then the equigeneric Severi variety $V_{g}(L)$ is pure of the expected dimension, see [8] and the references therein (this is stated here in Proposition 7). For a general such surface, it is also known that the nodal Severi variety is non-empty by [4] for $K 3$ 's and [10] for Abelian surfaces. The density of the nodal Severi variety in the equigeneric one was so far only known if in addition $L$ is primitive (and $g \geqslant 5$ in the Abelian case), see $[5,6]$ (as well as $[8,11]$ ) for the $K 3$ case, and [11] for the Abelian case.

Remark 3. On Enriques surfaces, it is proved in [7] that the irreducible components of the nodal Severi variety $V^{p(L)-g}(L)$ have dimension either $g-1$ or $g$. In the range of application of Theorem 1, there is only one component of dimension $g-1$, and the condition given in [7] to distinguish between the two cases tells us that for a general $[C] \in V^{p(L)-g}(L)$, the pull-back of $K_{S}$ to the normalization of $C$ is non-trivial.

As the main step in his proof, Ballico establishes the following statement. 
Proposition 4. Let $L$ be a line bundle on a smooth complex projective surface. If $L$ is very ample and $(2 \delta-1)$-spanned, then the family $\Sigma_{\delta}(L)$ of all members of $|L|$ which are singular in (at least) $\delta$ points is irreducible of codimension $\delta$ in $|L|$.

The result of Kool, Shende and Thomas that we use is the following, see [12, Prop. 2.1].

Proposition 5. Let $L$ be a line bundle on a smooth complex projective surface. If $L$ is $\delta$-very ample, then the general $\delta$-dimensional linear subsystem $\mathbf{P}^{\delta} \subseteq|L|$ contains a finite number of $\delta$-nodal curves, and all other members are reduced curves of geometric genus $p_{g}>p(L)-\delta$.

This has the following immediate corollary: in the setting of the proposition, if $V$ is an irreducible variety of codimension $\leqslant \delta$ in $|L|$ parametrizing curves of geometric genus $p_{g} \leqslant$ $p(L)-\delta$, then the general member of $V$ is in fact a $\delta$-nodal curve.

Corollary 6. If $L$ is $\delta$-very ample and $(2 \delta-1)$-spanned, then the Severi variety of nodal curves $V^{\delta}(L)$ is non-empty and irreducible of codimension $\delta$ in $|L|$.

Proof. Every irreducible component of $V^{\delta}(L)$ is contained in $\Sigma_{\delta}(L)$. On the other hand, $\Sigma_{\delta}(L)$ is irreducible of codimension $\delta$ by Prop. 4 , and has an open subset contained in $V^{\delta}(L)$ by Prop. 5 .

In the cases of Theorem 1, one has the following estimates on the dimensions of the Severi varieties.

Proposition 7. Let $S$ be a $K 3$ (resp. Enriques, resp. Abelian) surface, L an effective line bundle on $S$, and $g \leqslant p(L)$ an integer. Every irreducible component of the equigeneric Severi variety $V_{g}(L)$ has dimension $=g$ (resp. $\geqslant g-1$, resp. $\left.=g-2\right)$.

For Enriques surfaces, the estimate follows from [8, Lem. 2.3 and ineq. (2.6)]. For $K 3$ and Abelian surfaces a well known extra argument is needed, see [8, Prop. 4.5 and 4.13].

Proof of Theorem 1. As we have observed above, under the assumptions of Theorem 1, $L$ is actually $(2 p(L)-2 g-1)$-very ample, hence also $(p(L)-g)$-very ample, so that both Propositions 4 and 5 apply for $\delta=p(L)-g$. It follows that $V^{\delta}(L)$ is an irreducible, dense, non-empty, open subset of $\Sigma_{\delta}(L)$.

On the other hand, let $V$ be an irreducible component of $V_{g}(L)$. By Prop. 7, $V$ has codimension $\leqslant \delta$ in $|L|$. It thus follows from Prop. 5 that the general member of $V$ is a $\delta$-nodal curve, hence $V$ is contained, and actually dense in $\Sigma_{\delta}(L)$. This concludes the proof.

\section{References}

[1] E. Ballico, On the irreducibility of the severi variety of nodal curves in a smooth surface, Arch. Math. (2019), https://doi.org/10.1007/s00013-019-01349-y.

[2] M. Beltrametti, P. Francia and A. J. Sommese, On Reider's method and higher order embeddings, Duke Math. J. 58 (1989), no. 2, 425-439.

[3] M. Beltrametti and A. J. Sommese, Zero cycles and kth order embeddings of smooth projective surfaces, in Problems in the theory of surfaces and their classification (Cortona, 1988), Sympos. Math., XXXII, Academic Press, London, 1991, With an appendix by Lothar Göttsche, 33-48.

[4] X. Chen, Rational curves on K3 surfaces, J. Algebraic Geom. 8 (1999), no. 2, 245-278.

[5] _ _ A simple proof that rational curves on K3 are nodal, Math. Ann. 324 (2002), no. 1, 71-104.

[6] _ Nodal curves on K3 surfaces, New York J. Math. 25 (2019), 168-173. 
[7] C. Ciliberto, T. Dedieu, C. Galati and A. L. Knutsen, A note on severi varieties of nodal curves on enriques surfaces, arXiv:1811.06435, to appear in proc. Indam workshop "Birational Geometry and Moduli Spaces".

[8] T. Dedieu and E. Sernesi, Equigeneric and equisingular families of curves on surfaces, Publ. Mat. 61 (2017), no. 1, 175-212.

[9] A. L. Knutsen, On kth-order embeddings of K3 surfaces and Enriques surfaces, Manuscripta Math. 104 (2001), no. 2, 211-237.

[10] A. L. Knutsen and M. Lelli-Chiesa, Genus two curves on abelian surfaces, prepublication arXiv:1901.07603.

[11] A. L. Knutsen, M. Lelli-Chiesa and G. Mongardi, Severi varieties and Brill-Noether theory of curves on abelian surfaces, J. Reine Angew. Math. 749 (2019), 161-200.

[12] M. Kool, V. Shende and R. P. Thomas, A short proof of the Göttsche conjecture, Geom. Topol. 15 (2011), no. 1, 397-406.

[13] H. Terakawa, Higher order embeddings of algebraic surfaces of Kodaira dimension zero, Math. Z. 229 (1998), no. 3, 417-433.

Thomas Dedieu. Institut de Mathématiques de Toulouse ; UMR5219. Université de Toulouse ; CNRS. UPS IMT, F-31062 Toulouse Cedex 9, France. thomas.dedieu@math.univ-toulouse.fr 